\title{
Application of Cerebrospinal Fluid AT(N) Framework on the Diagnosis of AD and Related Cognitive Disorders in Chinese Han Population
}

This article was published in the following Dove Press journal: Clinical Interventions in Aging

\author{
Ling-Qi $\mathrm{Ye}^{1,2}$ \\ Pei-Rong Gao' \\ Yan-Bin Zhang ${ }^{1,3}$ \\ Hong-Rong Cheng ${ }^{1,4}$ \\ Qing-Qing Tao (D) \\ Zhi-Ying Wu' \\ Hong-Lei Li'
}

'Department of Neurology and Research Center of Neurology in Second Affiliated Hospital, and Key Laboratory of Medical Neurobiology of Zhejiang Province, Zhejiang University School of Medicine, Hangzhou, People's Republic of China;

${ }^{2}$ Department of Rehabilitation Medicine and Rehabilitation \& Sports Medicine Research Institute of Zhejiang Province, Zhejiang Provincial People's Hospital, People's Hospital of Hangzhou Medical College, Hangzhou, People's Republic of China; ${ }^{3}$ Department of Neurology and Institute of Neurology in First Affiliated Hospital, Fujian Medical University, Fuzhou, People's Republic of China; ${ }^{4}$ Department of Neurology in Fourth Affiliated Hospital, Zhejiang University School of Medicine, Yiwu, People's Republic of China
Background: Studies concerning the impact of the AT(N) framework on diagnostic capability in the dementia population are lacking. We aimed to explore the diagnostic application of CSF AT(N) framework in clinical routines of Alzheimer's disease (AD) as well as differential diagnosis of other cognitive diseases in the Chinese Han population.

Patients and Methods: A total of 137 patients with cognitive disorders received CSF tests of $\mathrm{A} \beta_{42}$, t-tau and $\mathrm{p}$-tau ${ }_{181}$. Their CSF biomarker results were categorized and interpreted by the AT(N) framework. Neurologists provided a diagnosis both pre- and post-CSF biomarker disclosure with corresponding diagnostic confidence.

Results: The total initial diagnosis included 79 patients with $\mathrm{AD}$ and 58 patients with nonAD (NAD). The results of CSF biomarkers led to a diagnostic change of $28 \%$ in the cohort. Approximately $81.5 \% \quad(n=53)$ of 65 patients whose CSF biomarker showed an underlying $\mathrm{AD}$ pathology were finally diagnosed as $\mathrm{AD}$, with an increase of $17.5 \%$ in diagnostic confidence. Thirty-seven CSF results indicating NAD pathologic changes contributed to an exclusion of $\mathrm{AD}$ in $56.8 \%(\mathrm{n}=21)$ of the patients along with a modest increase of $9.8 \%$ in average confidence. Thirty-

five patients with normal CSF biomarkers maintained the diagnosis of NAD in $68.6 \%(n=24)$ of the group, leading to a slight elevation of $7.6 \%$ in confidence. Conclusion: We found that the presence of amyloid pathology $(\mathrm{A}+)$ is contributable to diagnosing $\mathrm{AD}$ and improving confidence. On occasion of negative amyloid pathology (A-), with or without tau pathology, gaining uncertainty of the primary $\mathrm{AD}$ diagnosis would diminish the corresponding confidence. To the best of our knowledge, this is the first study performed in the Chinese Han population with cognitive disorders that explores the clinical capability of CSF AT(N) framework in a quantitative way.

Keywords: Alzheimer's disease, cerebrospinal fluid biomarker, AT(N), diagnostic confidence

\section{Introduction}

Currently, two pathological hallmarks of Alzheimer's disease (AD) are wellrecognized as toxic senile plaques formed by extracellular $\beta$-amyloid deposition and neurofibrillary tangles (NFTs) formed with intracellular accumulation of hyperphosphorylated tau in the brain. ${ }^{1}$ In most ante-mortem cases, the diagnosis of AD mainly depends on typical symptoms of cognitive decline, neuropsychological tests and neuroimaging examination. ${ }^{2}$ The present drug and non-drug therapies often fail to meet satisfactory response at an advanced stage of AD. Several clinical trials
Correspondence: Hong-Lei Li Department of Neurology and Research Center of Neurology in Second Affiliated Hospital, and Key Laboratory of Medical Neurobiology of Zhejiang Province, Zhejiang University School of Medicine, 88 jiefang Road, Hangzhou, 310009

People's Republic of China

Tel/Fax +86-57I-87783569

Email lihonglei@zju.edu.cn 
only show limited effects in patients at an early stage. Hence, early identification and diagnosis is the most critical prevention and management of AD. Nevertheless, comparing with the diagnostic accuracy in autopsy studies, the sensitivity and specificity is merely $71 \%$ for probable AD in clinical practice. ${ }^{1}$ Encountering conditions like younger patients (senile dementia), patients manifesting as atypical AD (non-amnestic symptoms such as visuospatial dysfunction, behavioral abnormality), comorbidity, or patients with a rapid progression make it harder to diagnose with sufficient confidence. ${ }^{3,4}$

Extensive evidence has confirmed that distinctive in-vivo biomarkers reflecting $\mathrm{AD}$ pathology could occur years or even decades before the clinical onset of dementia. The National Institute on Aging and Alzheimer's Association (NIA-AA) has proposed an AT(N) framework for $\mathrm{AD}$ research in recent years, ${ }^{5}$ which represents significant progress in the biological characterization of the disease spectrum of $\mathrm{AD}$. In the framework, " $\mathrm{A}$ " refers to amyloidosis, "T" stands for tauopathy, and "(N)" represents a non-specific biomarker of neurodegeneration or neuronal injury. Each biomarker category is binarized as positive $(+)$ or negative $(-)$, resulting in 8 different $\mathrm{AT}(\mathrm{N})$ profiles, ranging from a group where all biomarkers are negative (A-T-(N)-) to one where are all positive $(\mathrm{A}+\mathrm{T}+(\mathrm{N})+)$.

It has been verified that decreased levels of cerebrospinal fluid (CSF) $\beta$ amyloid protein with 42 amino acids $\left(\mathrm{A} \beta_{42}\right)$ and increased CSF total tau (t-tau) as well as phosphorylated isoform of tau (p-tau) directly reflect neuropathological changes in the brains of $\mathrm{AD}^{6}$ Combinations of the 3 CSF biomarkers have been widely promoted in clinical diagnosis of $\mathrm{AD},{ }^{7-9}$ prediction of rate of progression, ${ }^{10}$ evaluation of $\mathrm{AD}$-converting risk in patients with mild cognitive impairment $(\mathrm{MCI}),{ }^{11}$ or in preclinical individuals with $\mathrm{AD},{ }^{12,13}$ in addition, contributing to drug development and $\mathrm{AD}$ clinical trials. ${ }^{14}$ However, studies on how these CSF biomarkers influence the diagnostic process of $\mathrm{AD}$ via the $\mathrm{AT}(\mathrm{N})$ framework are limited, ${ }^{15,16}$ and it has never been examined in Chinese Han population. Therefore, our research aims to quantify the impact of CSF AT(N) framework on clinical diagnosis of $\mathrm{AD}$ as well as its differential diagnosis.

\section{Patients and Methods}

\section{Subjects and Diagnostic Criteria}

We consecutively enrolled a total of 137 patients with complaints of cognitive decline from the Department of
Neurology, Second Affiliated Hospital of Zhejiang University School of Medicine between March 2015 and October 2019. All participants received a standard screening procedure of dementia including collections of detailed medical history, physical and neurological examinations, basic neuropsychological examination, routine laboratory testing and brain magnetic resonance imaging (MRI). All examinations were conducted by experienced doctors and technicians. The patient would be diagnosed as dementia if he/she met the DSM-IV criteria of dementia. ${ }^{17}$ Demented participants would be further diagnosed with probable AD if they met NINCDS-ADRDA criteria. ${ }^{2}$ Patients who fulfilled the Neary criteria would be diagnosed with frontotemporal lobar dementia (FTD), ${ }^{18}$ vascular dementia $(\mathrm{VaD})$ if they met the NINCDS-AIREN criteria, ${ }^{19}$ dementia with Lewy bodies (DLB) if they met the criteria of McKeith et al, ${ }^{20}$ normal pressure hydrocephalus (NPH) if they met the revised Dandy criteria. ${ }^{21}$ According to different diagnostic criteria the patients fulfilled, other diagnoses including progressive supranuclear palsy (PSP), ${ }^{22}$ Parkinson's disease with dementia (PDD), ${ }^{23}$ and mixed dementia (AD-VaD) would be made. Patients who failed to meet the criteria of dementia but reached the criteria of Petersen et al would be diagnosed as MCI. ${ }^{24}$ At the beginning, two senior neurologists evaluated each participant with provided clinical data, neuropsychological assessment and MRI information. They determined initial diagnosis separately and rated corresponding diagnostic confidence (range, 50\%-100\%). When the diagnosis given by two neurologists were controversial, a final decision and corresponding confidence would be given after they reached an agreement.

\section{Collection of CSF Samples}

A lumbar puncture (LP) and CSF test would be requested whenever the clinicians were uncertain about the diagnosis. It was ensured that there were no contraindications to LP for each patient before it was performed. CSF samples were collected in a polypropylene tube by LP at L3/4 or L4/5 space and were transported immediately to laboratory. The samples were centrifuged at $400 \times \mathrm{g}$ for $10 \mathrm{~min}$ at $4^{\circ} \mathrm{C}$ to remove cells, then frozen and stored in Protein LoBind Tubes (Eppendorf AG, Germany) at $-80^{\circ} \mathrm{C}$ before analysis. A small portion of the CSF was used for routine laboratory testing. The results of total cells (leucocytes and erythrocytes), protein and glucose were unremarkable in all samples. 


\section{CSF Biomarker Analysis}

CSF A $\beta_{42}$, t-tau and tau phosphorylated at threonine-181 (p$\operatorname{tau}_{181}$ ) were later assessed by INNOTEST sandwich enzyme linked immunosorbent assays (ELISA) (Fujirebio, Ghent, Belgium) according to the manufacturer's instructions by experienced technicians who were blind to clinical diagnosis.

There are no international standardized cutoffs of these AD biomarkers yet, hence in this study we take the cutoffs listed on the ELISA instruction as reference, which are determined by an unpublished data of the comparison between autopsyconfirmed $\mathrm{AD}$ patients and cognitively normal elderly. ${ }^{25}$ Normal concentrations of CSF $A \beta_{42}$, t-tau and $\mathrm{p}$-tau ${ }_{181}$ are associated with age and are described in the following:

- For 21 to 51 years old, $\mathrm{CSF} A \beta_{42}<610 \mathrm{pg} / \mathrm{mL}$, t-tau $>225 \mathrm{pg} / \mathrm{mL}$;

- For 51 to 70 years old, $C S F A \beta_{42}<562 \mathrm{pg} / \mathrm{mL}$, t-tau $>370 \mathrm{pg} / \mathrm{mL}$;

- For over 70 years old, CSF $A \beta_{42}<567 \mathrm{pg} / \mathrm{mL}$, t-tau $>512 \mathrm{pg} / \mathrm{mL}$.

- For 18 to 44 years old, CSF p-tau ${ }_{181}>45.67 \mathrm{pg} / \mathrm{mL}$ and $>66.26 \mathrm{pg} / \mathrm{mL}$ between 45 to 77 years old.

According to the AT(N) framework, ${ }^{5}$ the CSF biomarker results are classified into $8 \mathrm{AT}(\mathrm{N})$ categories and thereby interpreted into 3 types of pathologies (Table 1). When only abnormal level of CSF $A \beta_{42}$ is present $(A+T$ $+(\mathrm{N})+, \mathrm{A}+\mathrm{T}+(\mathrm{N})-, \mathrm{A}+\mathrm{T}-(\mathrm{N})+, \mathrm{A}+\mathrm{T}-(\mathrm{N})-)$, "Alzheimer's continuum" is used as a generic term which is comprised of three pathological conditions including "AD pathology," "Alzheimer's and concomitant suspected nonAlzheimer's pathologic change," and "Alzheimer's pathologic change." Individuals with a biomarker category showing "Alzheimer's continuum" are at high risk of developing $\mathrm{AD}$ or probable $\mathrm{AD}$. When levels of CSF tau are abnormal while $\mathrm{CSF} A \beta_{42}$ is normal $(\mathrm{A}-\mathrm{T}+(\mathrm{N})+$, $\mathrm{A}-\mathrm{T}+(\mathrm{N})-$, A-T-(N)+), "Non-Alzheimer's disease (NAD) pathologic changes" is used. "Normal AD biomarker" is considered when all 3 biomarkers are normal (A-T-(N)-).

\section{Outcome Measures}

The neurologists revised the diagnosis and re-evaluated the corresponding confidence (range, $50 \%-100 \%$ ) in reference to CSF AT(N) framework. Based on the consistency of diagnosis both pre- and post-CSF biomarker disclosure, the total 137 patients were divided into 5 groups: Group Stable AD, Group Stable NAD, Group Change AD to NAD, Group Change
Table I Biomarker Profiles and Pathological Interpretations

\begin{tabular}{|c|c|c|}
\hline AT(N) Profiles & Biomarker Category & $\begin{array}{l}\text { Pathological } \\
\text { Interpretation }\end{array}$ \\
\hline $\mathrm{A}+\mathrm{T}+(\mathrm{N})+$ & Alzheimer's disease & \multirow{4}{*}{$\begin{array}{l}\text { Alzheimer's } \\
\text { continuum }^{\text {a }}\end{array}$} \\
\hline$A+T+(N)-$ & Alzheimer's disease & \\
\hline$A+T-(N)+$ & $\begin{array}{l}\text { Alzheimer's and } \\
\text { concomitant suspected } \\
\text { non-Alzheimer's } \\
\text { pathologic change }\end{array}$ & \\
\hline$A+T-(N)-$ & $\begin{array}{l}\text { Alzheimer's pathologic } \\
\text { change }\end{array}$ & \\
\hline $\mathrm{A}-\mathrm{T}+(\mathrm{N})+$ & $\begin{array}{l}\text { Non-AD pathologic } \\
\text { change }\end{array}$ & \multirow{3}{*}{$\begin{array}{l}\text { Non-Alzheimer's } \\
\text { disease } \\
\text { pathologic } \\
\text { changes }^{\text {b }}\end{array}$} \\
\hline$A-T+(N)-$ & $\begin{array}{l}\text { Non-AD pathologic } \\
\text { change }\end{array}$ & \\
\hline $\mathrm{A}-\mathrm{T}-(\mathrm{N})+$ & $\begin{array}{l}\text { Non-AD pathologic } \\
\text { change }\end{array}$ & \\
\hline A-T-(N)- & Normal AD biomarkers & $\begin{array}{l}\text { Normal AD } \\
\text { biomarker }{ }^{c}\end{array}$ \\
\hline
\end{tabular}

Notes: Each biomarker category is binarized as positive (+) or negative (-), resulting in eight different $A T(N)$ biomarker profiles; Every individual can be classified into one of the three categories based on biomarker profiles: those who are in the Alzheimer's continuum (dark grey), those with non-AD pathologic changes (light grey), and those with normal AD biomarkers (no color); ${ }^{\mathrm{W}}$ When only abnormal level of CSF $A \beta_{42}$ is present, the $A T(N)$ profile should be classified into the "Alzheimer's continuum" group, which is an umbrella term comprised of three pathological conditions including "AD pathology," "Alzheimer's and concomitant suspected nonAlzheimer's pathologic change," and "Alzheimer's pathologic change;" 'When levels of CSF tau are abnormal while CSF $A \beta_{42}$ was normal, the $A T(N)$ profile should be classified into the "Non-Alzheimer's disease pathologic changes" group; "When all 3 biomarkers are normal, the $A T(N)$ profile should be classified into the "Normal $A D$ biomarker" group.

Abbreviation: AD, Alzheimer's disease.

NAD to AD, Group Change NAD to NAD. They integrated as Group Final AD and Group Final NAD, respectively.

\section{Statistical Analysis}

The statistical analyses were performed using SPSS 24.0. Quantitative data were compared using $t$-test, OneANOVA and $X^{2}$ test for categorical data between groups. Post-hoc analysis was calculated by Mann-Whitney $U$-Tests when evaluating changes in diagnosis and confidence. Significance level was defined as $p<0.05$.

\section{Results}

\section{Demographic Characteristics and Clinical Data}

The initial diagnosis of all 137 participants included $79 \mathrm{AD}(58 \%)$ and 58 NAD (42\%). The AD group 
consisted of 71 patients with typical AD dementia, 5 with atypical $\mathrm{AD}$ dementia, and 3 with prodromal $\mathrm{AD}$. The NAD group was comprised of 15 individuals with MCI, 27 with FTD, 4 with NPH, 3 with VaD, 2 with DLB, 2 with mixed dementia, 2 with leukoencephalopathy, and PDD, PSP, pseudodementia caused by depression, one for each. Baseline demographic characteristics and clinical data of the study population were summarized in Table 2. Subjects with an initial diagnosis of AD showed no statistical significance from NAD patients in terms of sex, age, age at onset, duration of disease or education except for the MMSE score, which was lower in the AD group (13.3 $\pm 7.1, p<0.05)$. In regards of CSF $\mathrm{AD}$ biomarkers, the values of CSF t-tau and $p$-tau ta1 $_{181}$ were both higher in the AD group than that in the NAD group $(p<0.001$, $p<0.01$ ).

\section{AT(N) Profiles of CSF Biomarker}

At baseline, distributions of CSF biomarker AT(N) profiles were exhibited in Table 3. Among the overall 137 participants, 65 results were interpreted into Alzheimer's continuum, 37 into NAD pathologic change, and 35 were classified as normal AD biomarker. The Alzheimer's continuum category was comprised of 42 individuals diagnosed with Initial AD and 23 with Initial NAD. The NAD pathologic change category consisted of 27 Initial AD and 10 Initial NAD. Ten patients diagnosed with Initial AD

Table 2 Demographic Characteristic and Clinical Data at Baseline

\begin{tabular}{|l|c|c|c|}
\hline Initial Diagnosis & $\begin{array}{c}\text { AD } \\
(\mathbf{n}=\mathbf{7 9})\end{array}$ & $\begin{array}{c}\text { NAD } \\
(\mathbf{n}=\mathbf{5 8})\end{array}$ & $\begin{array}{c}\text { Total } \\
(\mathbf{n}=137)\end{array}$ \\
\hline Sex, Male\% & $49.4 \%$ & $50.6 \%$ & $48.2 \%$ \\
\hline Age, years & $62.2 \pm 9.9$ & $60.7 \pm 9.7$ & $61.6 \pm 9.8$ \\
\hline Age at onset, years & $\begin{array}{c}59.4 \pm \\
10.3\end{array}$ & $58.2 \pm 10.3$ & $58.9 \pm 10.2$ \\
\hline Duration, years & $2.9 \pm 2.4$ & $2.5 \pm 2.3$ & $2.7 \pm 2.3$ \\
\hline Education, years & $7.6 \pm 4.2$ & $7.5 \pm 3.7$ & $7.6 \pm 4.0$ \\
\hline MMSE score & $13.3 \pm 7.1$ & $17.2 \pm 8.9{ }^{\mathrm{a}}$ & $14.9 \pm 8.1$ \\
\hline CSF A $\beta_{42}(\mathrm{pg} / \mathrm{mL})$ & $619 \pm 268$ & $703 \pm 382$ & $655 \pm 322$ \\
\hline CSF t-tau $(\mathrm{pg} / \mathrm{mL})$ & $448 \pm 292^{\mathrm{b}}$ & $276 \pm 246$ & $375 \pm 286$ \\
\hline CSF p-tau ${ }_{181}(\mathrm{pg} / \mathrm{mL})$ & $72 \pm 44^{\mathrm{c}}$ & $51 \pm 33$ & $63 \pm 41$ \\
\hline
\end{tabular}

Notes: Data are presented as mean \pm S.D. or percentage (\%); ${ }^{a} A D<N A D$ $(p<0.01)$; ${ }^{\mathrm{b}} \mathrm{AD}>\mathrm{NAD}(p<0.001)$; ${ }^{\mathrm{C}} \mathrm{AD}>\mathrm{NAD}(p<0.01)$.

Abbreviations: AD, Alzheimer's disease; CSF, cerebrospinal fluid; MMSE, MiniMental State Examination; NAD, non-Alzheimer's disease.
Table 3 Distribution of Cerebrospinal Fluid Biomarker AT(N) Profiles in Groups

\begin{tabular}{|c|c|c|c|c|}
\hline $\begin{array}{l}\text { Biomarker } \\
\text { Category }\end{array}$ & $\begin{array}{l}\text { AT }(\mathbf{N}) \\
\text { Profile }\end{array}$ & $\begin{array}{c}\text { AD } \\
(n=79)\end{array}$ & $\begin{array}{l}\text { NAD } \\
(n=58)\end{array}$ & $\begin{array}{c}\text { Total } \\
(n=137)\end{array}$ \\
\hline \multirow{5}{*}{$\begin{array}{l}\text { Alzheimer's } \\
\text { continuum }\end{array}$} & $A+T+(N)+$ & 11 & 4 & 15 \\
\hline & $A+T+(N)-$ & 2 & 0 & 2 \\
\hline & $A+T-(N)+$ & 5 & 2 & 7 \\
\hline & $A+T-(N)-$ & 24 & 17 & 41 \\
\hline & Number (\%) & $\begin{array}{c}42 \\
(64.6)\end{array}$ & $\begin{array}{c}23 \\
(35.4)\end{array}$ & 65 \\
\hline \multirow{4}{*}{$\begin{array}{l}\text { NAD pathologic } \\
\text { change }\end{array}$} & $\mathrm{A}-\mathrm{T}+(\mathrm{N})+$ & 20 & 6 & 26 \\
\hline & $A-T+(N)-$ & 3 & I & 4 \\
\hline & $A-T-(N)+$ & 4 & 3 & 7 \\
\hline & Number (\%) & $\begin{array}{c}27 \\
(73.0)\end{array}$ & $\begin{array}{c}10 \\
(27.0)\end{array}$ & 37 \\
\hline \multirow{2}{*}{$\begin{array}{l}\text { Normal AD } \\
\text { biomarker }\end{array}$} & A-T-(N)- & 10 & 25 & 35 \\
\hline & Number (\%) & $\begin{array}{c}10 \\
(28.6)\end{array}$ & $\begin{array}{c}25 \\
(71.4)\end{array}$ & 35 \\
\hline
\end{tabular}

Note: Data expressed as integral numbers and percentage (exhibited with brackets, the respective proportion of $A D$ group and NAD group in the total participants).

Abbreviations: AD, Alzheimer's disease; NAD, non-Alzheimer's disease.

and 25 with Initial NAD made up the normal category. In the Initial AD group ( $\mathrm{n}=79$ ), Alzheimer's continuum and NAD pathologic change accounted for 53\% and 34\%, respectively. Comparatively, in the Initial NAD group $(\mathrm{n}=58)$, Alzheimer's continuum and normal AD biomarker respectively took up a close proportion of $40 \%$ and $43 \%$ whereas NAD pathologic change was merely $17 \%(n=10)$. Generally, Alzheimer's continuum made up the most represented category (47\%) of the entire 137 subjects. The share of the other 2 categories almost halved the other $53 \%$.

\section{Revision in Clinical Diagnosis}

Given CSF biomarker AT(N) profile, 28\% of the diagnoses changed after biomarker disclosure (Figure 1). Among 79 individuals diagnosed as Initial AD, approximately $27 \%(\mathrm{n}=21)$ were revised into NAD, including 6 individuals with mixed dementia, 4 with $\mathrm{VaD}, 4$ with FTD, 2 with leukoencephalopathy, 4 with dementia of unknown etiology, and one pseudodementia caused by depression. As for the Initial NAD group, about $28 \%$ $(n=16)$ of the diagnosis changed into $\mathrm{AD}$ and one $\mathrm{VaD}$ 


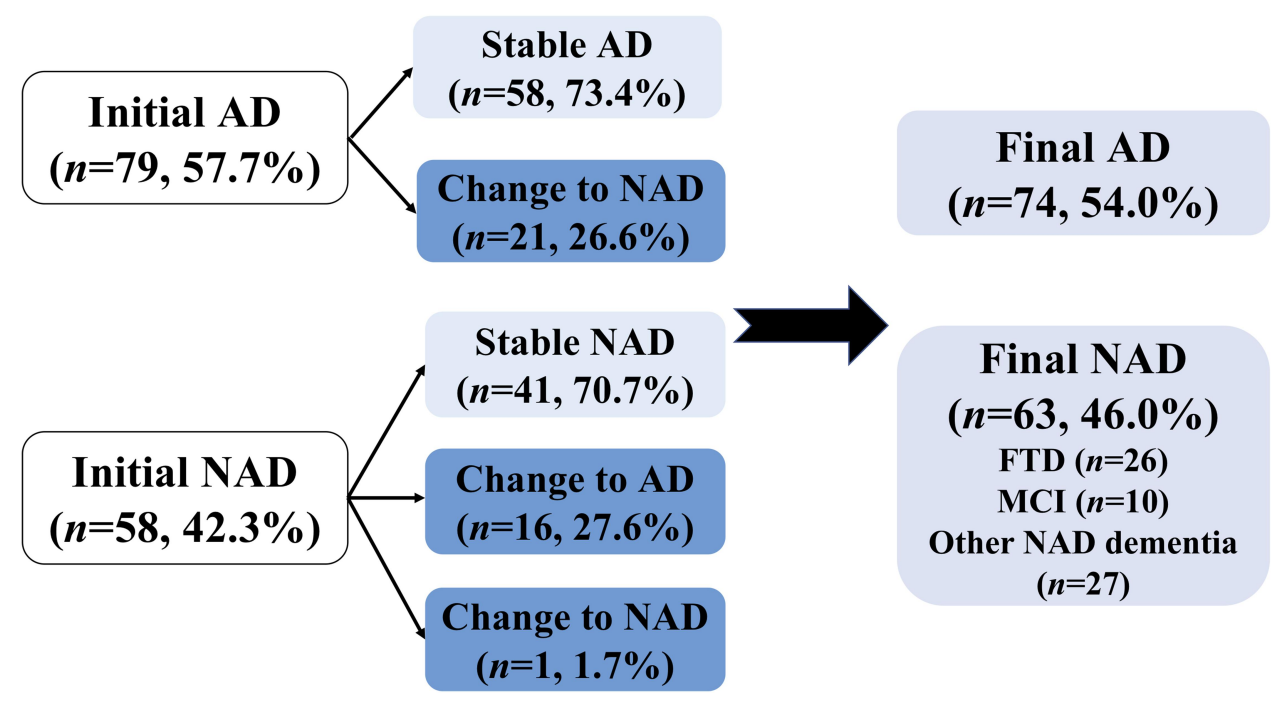

Figure I Revision of initial diagnosis post-CSF biomarker disclosure based on the AT(N) framework.

diagnosis was revised to mixed dementia. Further analysis (Figure 2A) revealed that the Alzheimer's continuum category made up the majority of both Group Stable AD $(\mathrm{n}=38,66 \%)$ and Group Change NAD to AD $(n=15$, 94\%). Normal AD biomarker category was predominant in Group Stable NAD $(n=24,59 \%)$ in contrast to the other 2 categories. With respect to Group Change AD to NAD, $52 \%$ of the CSF results $(n=11)$ showed NAD pathologic change, 29\% showed Alzheimer's continuum, and the remaining $19 \%$ belonged to normal $\mathrm{AD}$ biomarker category. Illustrated from Figure $2 \mathrm{~B}$ and $\mathrm{C}$, the Final AD group ( $\mathrm{n}=74$ ) was comprised of 53 individuals with Alzheimer's continuum (72\%), 16 with NAD pathologic change $(21 \%)$, and 5 with normal AD biomarker (7\%). Meanwhile, the 3 categories took up 19\%, 33\% and $48 \%$ in the Final NAD group ( $\mathrm{n}=63)$, respectively.

\section{Impact of CSF AT(N) Profile on Diagnostic Confidence}

An overview of the impact of CSF AT(N) framework on diagnostic confidence in 5 diagnostic subgroups as well as Group Final AD and Group Final NAD was exhibited in Table 4. Among the 5 diagnostic subgroups, CSF AT(N) profiles had the greatest effect on confidence in Group Change NAD to AD, which increased by $18.5 \pm 8.3 \%$. As described in Table 4, the post-CSF confidence was evidently comparable with the pre-CSF confidence in Group Stable AD, Group Stable NAD and Group Change NAD to $\mathrm{AD}(p<0.0001 ; p<0.0001 ; p<0.0001)$, whereas no significant differences were found in the other two groups.
On the whole, after given CSF AT(N) profiles the average diagnostic confidence increased from $70.6 \%$ to $80.4 \%$.

More specifically, $81.5 \%$ of 65 individuals with CSF profiles of Alzheimer's continuum were finally diagnosed as $\mathrm{AD}$ (Group Stable AD ( $\mathrm{n}=38)$ and Group Change NAD to $\mathrm{AD}(\mathrm{n}=15))$, showing a remarkable leap in the diagnostic confidence post-CSF disclosure $(p<0.0001 ; p<0.0001)$ (Figure 3A). On the other hand, in 37 individuals whose CSF profiles indicating NAD pathologic change, Group Stable NAD (11.5\%) and Group Change AD to NAD $(8.3 \%)$ both exhibited a moderate rise in confidence, which was lower than that in Alzheimer's continuum (Figure 3B). About $68.6 \%$ of the individuals with normal AD biomarker maintained the diagnosis of NAD and the confidence increased by $10.7 \%$. Interestingly, the post-CSF confidence exhibited apparent descending trends compared to pre-CSF confidence in individuals with consistent diagnosis of $\mathrm{AD}$ and those who were revised as NAD (Figure 3C).

It was apparent that Alzheimer's continuum contributed most to individuals with a final diagnosis of $\mathrm{AD}$ (Figure 4A), reaching an average increase of $17.5 \%$ in confidence and a post-CSF confidence of $88.8 \%$. The rising confidence overwhelmed the changes caused by the other two categories. On the contrary, as displayed in Group Final NAD (Figure 4B), NAD pathologic changes and Normal AD biomarker seemed to play more an important role in raising diagnostic confidence than Alzheimer's continuum $(p<0.001 ; p<0.05)$. It was demonstrated that the post-CSF confidence had a significant elevation compared to pre-CSF confidence in both Group Final AD and Group 

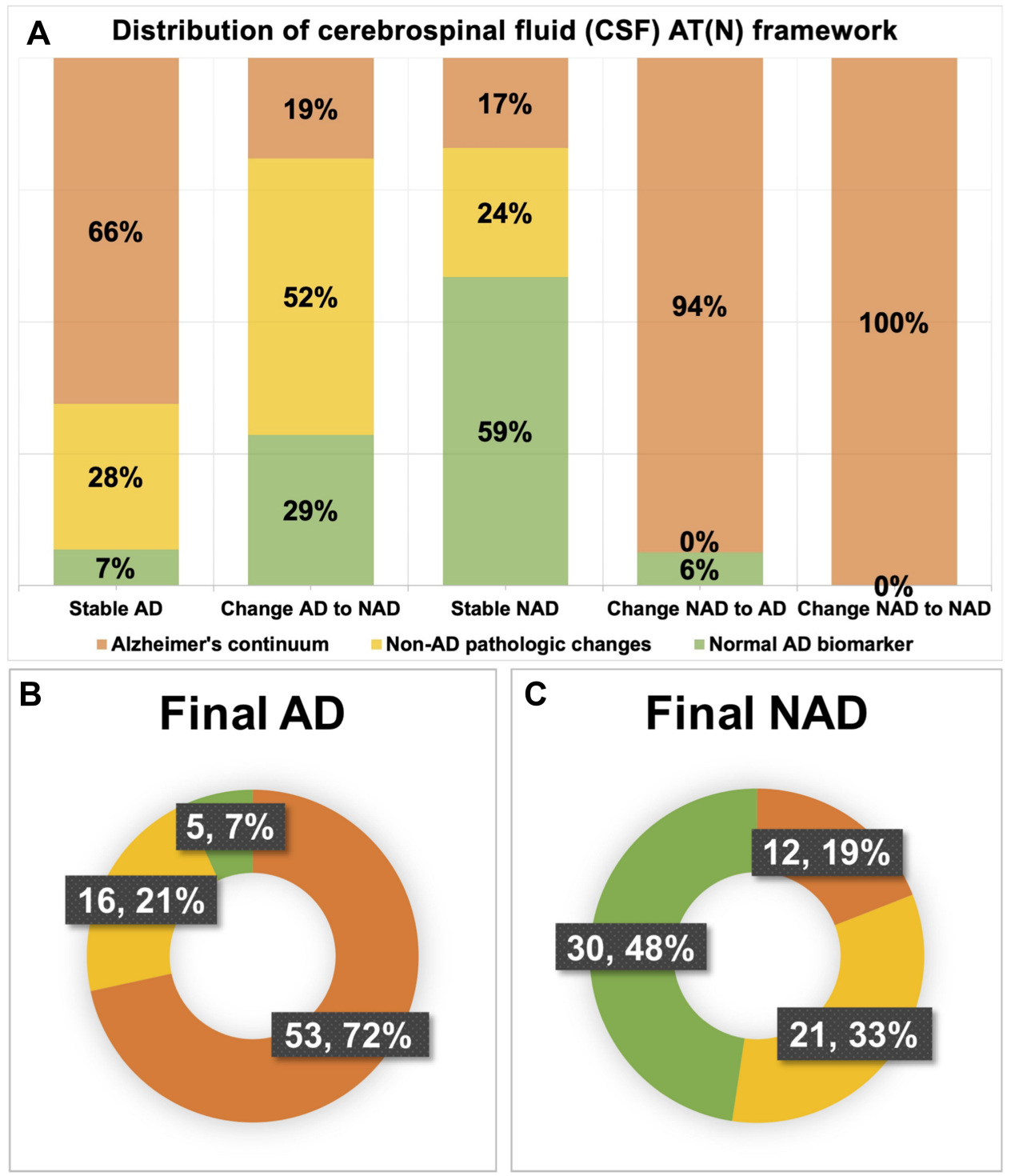

C

\section{Final NAD}

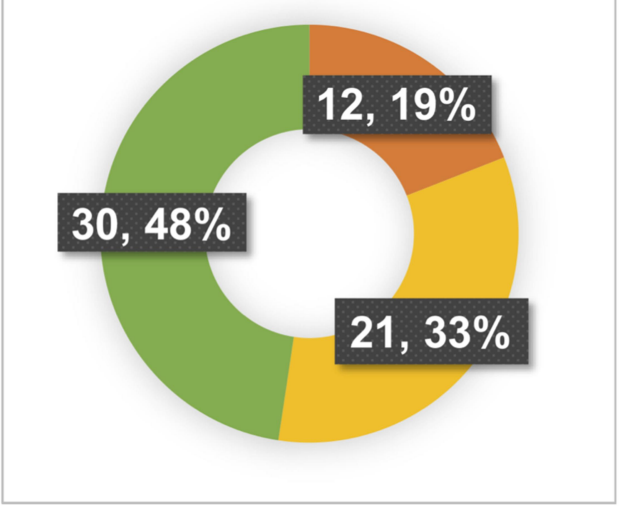

Figure 2 Distribution of cerebrospinal fluid (CSF) biomarker profiles based on the $A T(N)$ framework in different diagnostic groups. (A) Distribution in five diagnostic subgroups: Group Stable AD, Group Change AD to NAD, Group Change NAD to AD, Group Stable NAD, Group Change NAD to NAD; (B) Distribution in Group Final AD; (C) Distribution in Group Final NAD. Notes: Alzheimer's continuum (orange), Non-AD pathologic changes (yellow), normal AD biomarkers (light green).

Final NAD $(p<0.0001 ; p<0.0001)$ (Figure 4C). Taken altogether, these results indicated that disclosure of CSF AT(N) profiles could have remarkable influence on the diagnostic process of $\mathrm{AD}$ and other related cognitive disorders.

\section{Discussion}

In the present study, the prospective design with clinical and biochemical interpretation made possible realistic evaluation of the impact of CSF AT(N) framework in Chinese Han population with cognitive disorders. About $28 \%$ of the primary diagnoses were changed post-CSF biomarker disclosure. We found that knowledge of clinical data and positive amyloid pathology $(\mathrm{A}+)$ are contributable to a more confident diagnosis of probable AD. On occasion of negative amyloid pathology (A-), with or without tau pathology, clinicians are more likely to exclude the possibility of $\mathrm{AD}$ or lower the corresponding confidence of primary AD diagnosis. However, it fails to provide more diagnostic clues of other types of dementia.

Prior investigations have demonstrated that CSF biomarker results are consistent with clinical diagnosis in about two thirds of the AD patients. ${ }^{8,26}$ In our cohort, the matching proportion was approximately $72 \%$, which was moderately higher. A similar investigation enrolling 109 participants conducted by Kester 


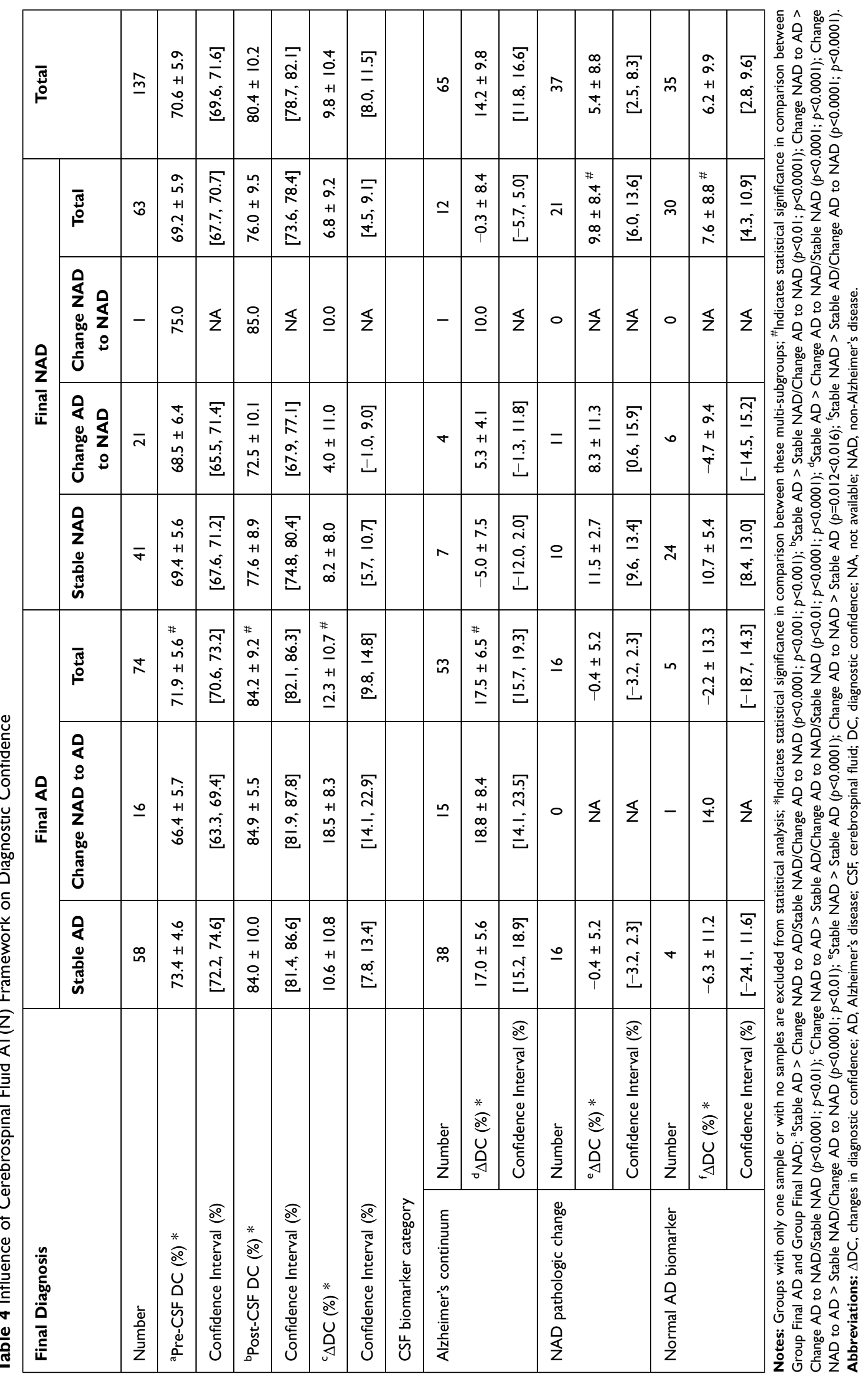




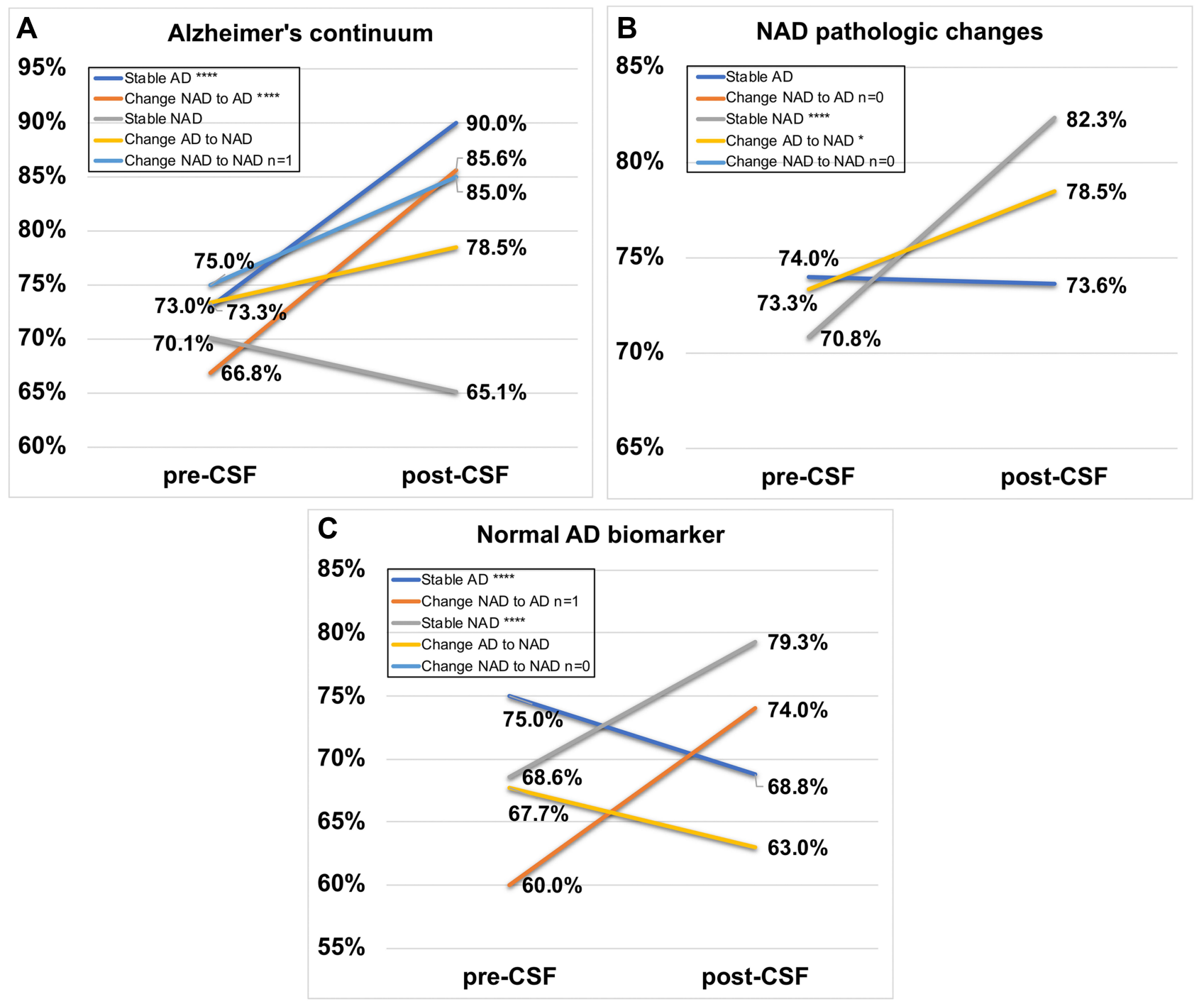

Figure 3 Different changes in diagnostic confidence post-CSF AT(N) profile disclosure compared to pre-CSF disclosure in five diagnostic subgroups. (A) Alzheimer's continuum; (B) Non-AD pathologic change; (C) Normal AD biomarkers. Notes: Group Stable AD (dark blue line), Group Change NAD to AD (orange line), Group Stable NAD (grey line), Group Change AD to NAD (yellow line), Group Change NAD to NAD (light blue line). “*” and “*****" stand for $p<0.05$ and $p<0.000$ I, respectively.

et al showed that the CSF biomarker disclosure only changed $10 \%$ of the diagnosis. ${ }^{27}$ Another large-scale multicenter CSF study involving 561 patients performed by Mouton-Liger et al exhibited a diagnostic change of $26.7 \%{ }^{16}$ Similar to the latter finding, our result revealed a diagnostic change of $28 \%$. The discrepancy in different studies is possibly to be influenced by sampling time, clinical data and clinical experience. Generally, the overall diagnostic confidence of the patients finally diagnosed with $\mathrm{AD}$ all showed an ascending trend. Kester et al found that the confidence in diagnosis elevated from $51 \%$ to $73 \%$ in reference to CSF biomarker profiles. ${ }^{27}$ Another small-scale study reported an increase in confidence from $77.4 \%$ to $86.2 \%{ }^{28}$ Close to the latter finding, the confidence of Group Final AD $(n=74)$ in our study increased from $71.9 \%$ to $84.2 \%$. Considering these different findings, we supposed that the umbrella term, Alzheimer's continuum, may influence the diagnostic decision to some extent because the presence of $\mathrm{A} \beta$ pathology incorporates a larger disease spectrum due to AD. Notably, a portion of the AD participants in this study were younger than the prevalent age of $\mathrm{AD}$ in Chinese Han population. One reason for this age bias was because younger patients were more willing to receive lumbar puncture in clinical practice. Another reason is that old Chinese patients are not willing to accept lumbar puncture for it is an invasive 


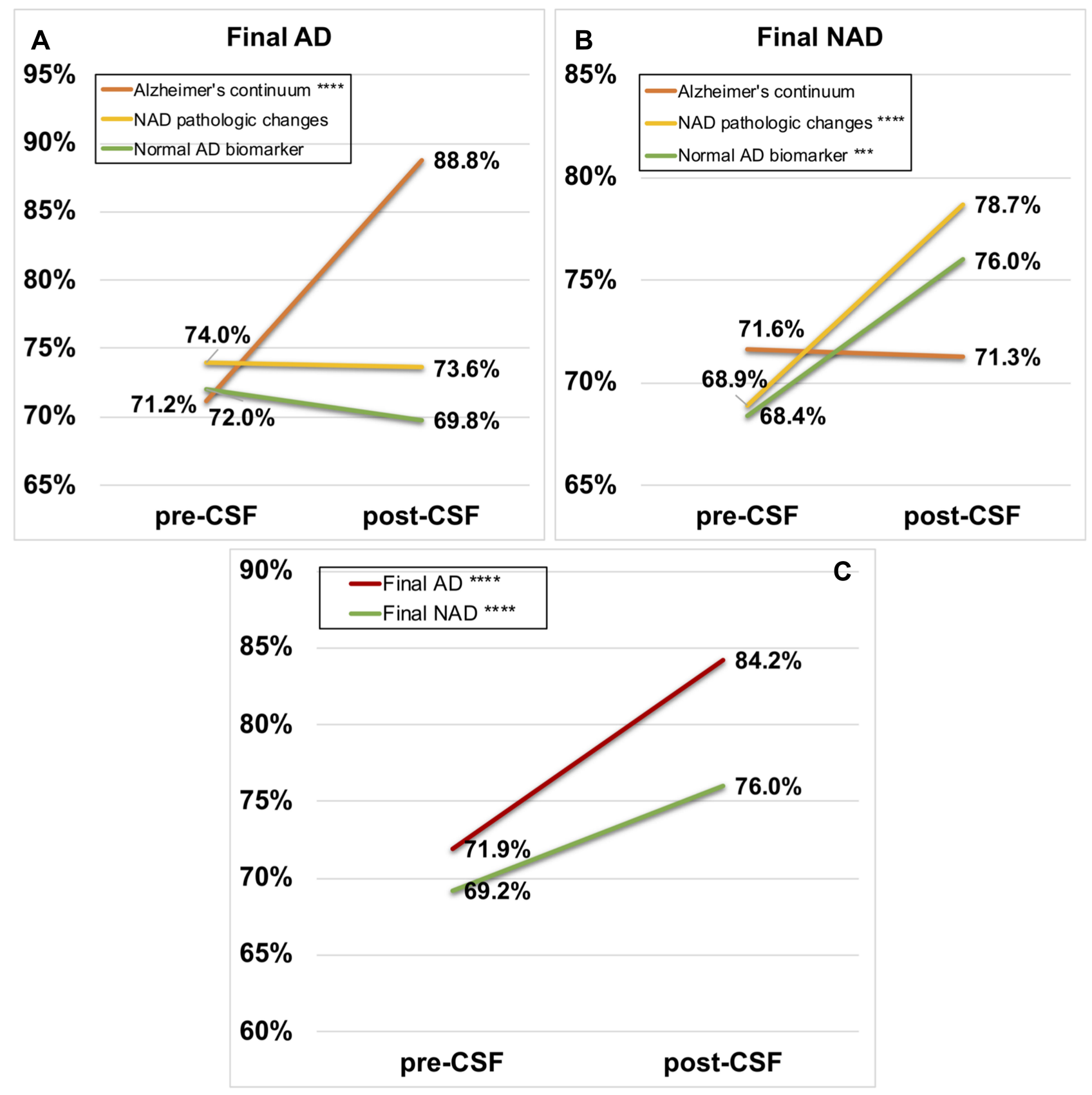

Figure 4 Different changes in diagnostic confidence due to three pathological interpretations of the AT(N) framework and total changes both in Group Final AD and Group Final NAD. In the upper two boxes: Alzheimer's continuum (orange line), Non-AD pathologic changes (yellow line), Normal AD biomarker (light green line). In the lower box: Final AD (red line), Final NAD (dark green line). (A) Group Final AD; (B) Group Final NAD; (C) Total participants. “***” and “*****” stand for $p<0.00$ I and $p<0.000$ I, respectively.

examination. It would be interesting to study how CSF biomarkers aid in the diagnosis of early-onset $\mathrm{AD}$ cases $(<65$ years). Since the populations of some of the previous works seem to be older and thus more representative of typical $\mathrm{AD}$, our result may be less representative because we did not specify any age range in this study. Nevertheless, this is only a preliminary study and further large-scale study with stratification analysis of each age distribution is required in the future.

Speaking of the cutoff values for CSF AD biomarkers, it should be suggested to use age-adjusted cutoff values. We reviewed previous literatures on cutoff values of CSF AD biomarkers between $\mathrm{AD}$ and controls and noticed that the values vary in populations and centers and largely depend on the sample size. ${ }^{29-32}$ Such CSF studies are less 
conducted in Asian populations and are often hampered by small sample sizes, ${ }^{33,34}$ generally speaking, yet there are no unified standard cutoff values for CSF AD biomarkers in Chinese Han population. Therefore, we chose the wellestablished Belgium cutoff values to classify the results. Furthermore, the fact that biomarker values are associated with age is also connected to the fact that $\mathrm{AD}$ onset is associated to age, which raises great importance in using age-adjusted cutoff values for CSF AD biomarkers so as to define the CSF biomarker results in a more reasonable and representative way.

Our study showed that $81.5 \%$ of the patients with Alzheimer's pathology were considered AD and the CSF biomarker results raised an increase of $17.5 \%$ in diagnostic confidence, which highlighted the critical role of amyloidosis in the $\mathrm{AD}$ pathogenesis. In the absence of direct pathologic evidence, biomarkers indicating Alzheimer's pathology significantly increase the confidence of $\mathrm{AD}$ diagnosis. In the other $18.5 \%$ of the NAD patients, the general diagnostic confidence was hardly affected. Previous findings showed that $\mathrm{AD}$ pathologic changes may also occur in cases of FTD, DLB, VaD and corticobasal degeneration (CBD). ${ }^{8}$ The overlapping AD pathologic change may be caused by mixed pathologies, particularly common in elder patients. ${ }^{35}$ For instance, some pathological research confirmed amyloidosis in brains of severe DLB patients, ${ }^{36-38}$ while senile plaques and NFTs in brains of progressive non-fluent aphasia (PNFA) patients were also observed, ${ }^{39}$ which verified the role of amyloid protein and phosphorylated tau in the pathogenesis of other types of dementia. ${ }^{40,41}$ Therefore, the presence of mixed pathology should be considered when encountering atypical cases or elder patient, which requires more clues to diagnose or exclude $\mathrm{AD}$.

When the CSF biomarkers exhibit as merely nonspecific neurodegeneration pathology with or without phosphorylated tau, the diagnostic confidence of NAD increases. About $56.8 \%$ of the 37 patients with CSF biomarkers indicating NAD pathologic change were finally diagnosed with NAD. The confidence increased by $9.8 \%$ on the whole. Especially in cases that changed from $\mathrm{AD}$ to NAD, the confidence mildly increased from $67.2 \%$ to $75.5 \%$. In patients with uncertain diagnosis of AD, CSF biomarkers lacking amyloid pathology are likely to assist in revising instead of maintaining the original diagnosis. On the contrary, in patients with more confident diagnosis of $\mathrm{AD}$, while maintaining the diagnosis, the mismatch in
CSF results and clinical data would lead to the decrease in confidence.

The normal AD biomarker category contributes to maintaining the diagnosis of NAD. About $68.6 \%$ of the total 35 patients with negative CSF biomarker results kept the original diagnosis and the confidence increased by $10.7 \%$. Meanwhile, the average confidence of patients with Initial AD who kept their original diagnosis reduced from $75.0 \%$ to $68.8 \%$ in reference to normal $\mathrm{AD}$ biomarkers. In general, evidence of normal AD biomarker only helps to exclude the possibility of an $\mathrm{AD}$ diagnosis whereas it cannot provide clues of other types of dementia. Hence, the lowered diagnostic confidence due to normal CSF results could remind clinicians to adjust diagnostic and therapeutic strategies.

Nevertheless, there were some limitations in our study. First of all, level of confidence is a subjective reflection of the opinions and behaviors due to clinicians, the value of which could be biased by personal knowledge and clinical experience. However, a definite diagnosis of dementia case is rare in clinical practice. Numerical confidence could bring ecologically objective data to quantify diagnostic certainty and provide therapeutic strategies that are available for $\mathrm{AD}$ as well as discriminating from other types of dementia. Besides, advanced evidence shows that the CSF $\mathrm{A} \beta_{42} / \mathrm{A} \beta_{40}$ ratio is considered a more reliable marker of brain amyloidosis. ${ }^{42}$ However, we did not include this candidate marker in the present study. This promising CSF biomarker is probably more valuable than CSF $\mathrm{A} \beta_{42}$ alone in the routine diagnostics of $\mathrm{AD}$. Further studies in regards of the diagnostic impact of the CSF $A \beta_{42} / A \beta_{40}$ ratio as well as other surrogate biomarkers are expected, such as blood biomarkers (blood $\mathrm{A} \beta$, blood p-tau and t-tau, neurofilament, $A \beta$ oligomerizations). In addition, although the total sample of AD patients and individuals with related cognitive disorders is relatively large in the present study, the numbers of respective NAD dementia are not big enough to compare with AD. This limits the categorized discussion of how CSF AT(N) framework distinguished $\mathrm{AD}$ from related cognitive disorders. We expect a larger sample size to be further analyzed. There is another technical limitation of the study. We used manual INNOTEST ELISA to test CSF biomarkers, which is a non-automated assay. This method suffers from relatively high (kit and operator) variability and therefore the use of the cut-points provided by the manufacturer might result in decreased performance. Since automated platforms such as Lumipulse (Fujirebio) provide better 
reproducibility by minimizing the preanalytical steps, we are considering taking the advanced method to improve the performance in future studies.

\section{Conclusion}

To the best of our knowledge, our work provides the first comprehensive assessment of the diagnostic capability of CSF AT(N) framework in Chinese Han population with cognitive disorders. Our results imply that using CSF $\mathrm{AT}(\mathrm{N})$ framework can assist clinicians in increasing confidence and differential diagnosis in AD. In general, the added value of CSF biomarkers in early diagnosis and effective intervention for $\mathrm{AD}$ are required to be further investigated.

\section{Abbreviations}

$\mathrm{A} \beta_{42}, \beta$ amyloid protein with 42 amino acids; $\mathrm{AD}$, Alzheimer's disease; CBD, cortico-basal degeneration; CSF, cerebrospinal fluid; DLB, dementia with Lewy bodies; DSM-IV, the fourth edition of Diagnostic and Statistical Manual of Mental Diseases; ELISA, enzyme linked immunosorbent assays; FTD, frontotemporal dementia; HD, Huntington's disease; LP, lumbar puncture; MCI, mild cognitive impairment; MMSE, Mini-Mental State Examination; MRI, magnetic resonance imaging; NAD, non-Alzheimer's disease; NFTs, neurofibrillary tangles; NIA-AA, the National Institute on Aging and Alzheimer's Association; NPH, normal pressure hydrocephalus; p-tau 181 , tau phosphorylated at threonine 181; PDD, Parkinson's disease with dementia; PNFA, progressive non-fluent aphasia; PSP, progressive supranuclear palsy; t-tau, total tau; VaD, vascular dementia.

\section{Data Sharing Statement}

The datasets generated and analyzed during the present study are available in the dementia database from Department of Neurology and Research Center of Neurology in the Second Affiliated Hospital, Zhejiang University School of Medicine.

\section{Ethics Approval and Informed Consent}

The study was approved by the Ethics Committee of the Second Affiliated Hospital of Zhejiang University School of Medicine. Written informed consents were obtained from the patients or their caregivers. We confirm that this study was conducted in accordance with the Declaration of Helsinki.

\section{Acknowledgments}

We are grateful to all participants for their willingness to participate in this study.

\section{Funding}

This study was supported by the National Natural Science Foundation of China to Hong-Lei Li [grant number $81500908]$.

\section{Disclosure}

All authors declare that there are no conflicts of interests.

\section{References}

1. Beach TG, Monsell SE, Phillips LE, Kukull W. Accuracy of the clinical diagnosis of Alzheimer disease at National Institute on Aging Alzheimer Disease Centers, 2005-2010. J Neuropathol Exp Neurol. 2012;71(4):266-273. doi:10.1097/NEN.0b013e31824b211b

2. McKhann G, Drachman D, Folstein M, Katzman R, Price D, Stadlan EM. Clinical diagnosis of Alzheimer's disease: report of the NINCDS-ADRDA work group under the auspices of Department of health and human services task force on Alzheimer's Disease. Neurology. 1984;34(7):939-944. doi:10.1212/WNL.34.7.939

3. Grundman M, Pontecorvo MJ, Salloway SP, et al. Potential impact of amyloid imaging on diagnosis and intended management in patients with progressive cognitive decline. Alzheimer Dis Assoc Disord. 2013;27(1):4-15. doi:10.1097/WAD.0b013e318279d02a

4. Wolk DA, Price JC, Madeira C, et al. Amyloid imaging in dementias with atypical presentation. Alzheimers Dement. 2012;8(5):389-398. doi:10.1016/j.jalz.2011.07.003

5. Jack CR, Bennett DA, Blennow K, et al. NIA-AA research framework: toward a biological definition of Alzheimer's disease. Alzheimers Dement. 2018;14(4):535-562. doi:10.1016/j. jalz.2018.02.018

6. Tapiola T, Alafuzoff I, Herukka SK, et al. Cerebrospinal fluid \{beta\}-amyloid 42 and tau proteins as biomarkers of Alzheimer-type pathologic changes in the brain. Arch Neurol. 2009;66(3):382-389. doi:10.1001/archneurol.2008.596

7. Lewczuk P, Riederer P, O'Bryant SE, et al. Cerebrospinal fluid and blood biomarkers for neurodegenerative dementias: an update of the consensus of the task force on biological markers in psychiatry of the world federation of societies of biological psychiatry. World $J$ Biol Psychiatry. 2018;19(4):244-328. doi:10.1080/15622975.2017.1375556

8. Schoonenboom NS, Reesink FE, Verwey NA, et al. Cerebrospinal fluid markers for differential dementia diagnosis in a large memory clinic cohort. Neurology. 2012;78(1):47-54. doi:10.1212/ WNL.0b013e31823ed0f0

9. Bjerke M, Engelborghs S. Cerebrospinal fluid biomarkers for early and differential Alzheimer's disease diagnosis. J Alzheimers Dis. 2018;62(3):1199-1209. doi:10.3233/JAD-170680

10. Kester MI, van der Vlies AE, Blankenstein MA, et al. CSF biomarkers predict rate of cognitive decline in Alzheimer disease. Neurology. 2009;73(17):1353-1358. doi:10.1212/WNL.0b013e3181bd8271

11. Wolfsgruber S, Polcher A, Koppara A, et al. Cerebrospinal fluid biomarkers and clinical progression in patients with subjective cognitive decline and mild cognitive impairment. $J$ Alzheimers Dis. 2017;58(3):939-950. doi:10.3233/JAD-161252 
12. Steenland K, Zhao L, Goldstein F, Cellar J, Lah J. Biomarkers for predicting cognitive decline in those with normal cognition. $J$ Alzheimers Dis. 2014;40(3):587-594. doi:10.3233/JAD-2014131343

13. Fagan AM, Roe CM, Xiong C, Mintun MA, Morris JC, Holtzman DM. Cerebrospinal fluid tau/beta-amyloid(42) ratio as a prediction of cognitive decline in nondemented older adults. Arch Neurol. 2007;64(3):343-349. doi:10.1001/archneur.64.3.noc60123

14. Cummings J. The role of biomarkers in Alzheimer's disease drug development. Adv Exp Med Biol. 2019;1118:29-61.

15. Duits FH, Prins ND, Lemstra AW, et al. Diagnostic impact of CSF biomarkers for Alzheimer's disease in a tertiary memory clinic. Alzheimers Dement. 2015;11(5):523-532. doi:10.1016/j. jalz.2014.05.1753

16. Mouton-Liger F, Wallon D, Troussière AC, et al. Impact of cerebrospinal fluid biomarkers of Alzheimer's disease in clinical practice: a multicentric study. J Neurol. 2014;261(1):144-151. doi:10.1007/ s00415-013-7160-3

17. American Psychiatric Association. DSM-IV: Diagnostic and Statistical Manual of Mental Disorders. 4th ed. American Psychiatric Association: Washington; 1994.

18. Neary D, Snowden JS, Gustafson L, et al. Frontotemporal lobar degeneration: a consensus on clinical diagnostic criteria. Neurology. 1998;51(6):1546-1554. doi:10.1212/WNL.51.6.1546

19. Román GC, Tatemichi TK, Erkinjuntti T, et al. Vascular dementia: diagnostic criteria for research studies. Report of the NINDS-AIREN International Workshop. Neurology. 1993;43(2):250-260. doi:10.1212/WNL.43.2.250

20. McKeith IG, Dickson DW, Lowe J, et al. Diagnosis and management of dementia with Lewy bodies: third report of the DLB Consortium. Neurology. 2005;65(12):1863-1872. doi:10.1212/01. wnl.0000187889.17253.b1

21. Friedman DI, Liu GT, Digre KB. Revised diagnostic criteria for the pseudotumor cerebri syndrome in adults and children. Neurology. 2013;81(13):1159-1165. doi:10.1212/WNL.0b013e3182a55f17

22. Höglinger GU, Respondek G, Stamelou M, et al. Clinical diagnosis of progressive supranuclear palsy: the movement disorder society criteria. Movement Disord. 2017;32(6):853-864. doi:10.1002/ mds. 26987

23. Hansen D, Ling H, Lashley T, et al. Novel clinicopathological characteristics differentiate dementia with Lewy bodies from Parkinson's disease dementia. Neuropathol Appl Neurobiol. 2020.

24. Petersen RC, Smith GE, Waring SC, Ivnik RJ, Tangalos EG, Kokmen E. Mild cognitive impairment: clinical characterization and outcome. Arch Neurol. 1999;56(3):303-308. doi:10.1001/ archneur.56.3.303

25. Van der Mussele S, Fransen E, Struyfs H, et al. Depression in mild cognitive impairment is associated with progression to Alzheimer's disease: a longitudinal study. $J$ Alzheimers Dis. 2014;42 (4):1239-1250. doi:10.3233/JAD-140405

26. Lim A, Tsuang D, Kukull W, et al. Clinico-neuropathological correlation of Alzheimer's disease in a community-based case series. $\mathrm{J} \mathrm{Am}$ Geriatr Soc. 1999;47(5):564-569. doi:10.1111/j.1532-5415.1999. tb02571.x

27. Kester MI, Boelaarts L, Bouwman FH, et al. Diagnostic impact of CSF biomarkers in a local hospital memory clinic. Dement Geriatr Cogn Disord. 2010;29(6):491-497. doi:10.1159/000313534
28. Falgàs N, Tort-Merino A, Balasa M, et al. Clinical applicability of diagnostic biomarkers in early-onset cognitive impairment. Eur J Neurol. 2019;26(8):1098-1104. doi:10.1111/ene.13945

29. Somers C, Struyfs H, Goossens J, et al. A decade of cerebrospinal fluid biomarkers for Alzheimer's disease in Belgium. J Alzheimers Dis. 2016;54(1):383-395. doi:10.3233/JAD-151097

30. Niemantsverdriet E, Goossens J, Struyfs H, et al. Diagnostic impact of cerebrospinal fluid biomarker (pre-)analytical variability in Alzheimer's disease. J Alzheimers Dis. 2016;51(1):97-106. doi:10.3233/JAD-150953

31. Mulder C, Verwey NA, van der Flier WM, et al. Amyloid-beta(1-42), total tau, and phosphorylated tau as cerebrospinal fluid biomarkers for the diagnosis of Alzheimer disease. Clin Chem. 2010;56 (2):248-253. doi:10.1373/clinchem.2009.130518

32. Mattsson N, Zetterberg H, Hansson O, et al. CSF biomarkers and incipient Alzheimer disease in patients with mild cognitive impairment. JAMA. 2009;302(4):385-393. doi:10.1001/ jama.2009.1064

33. Liu B, Tang Y, Shen Y, Cen L, Han M. Cerebrospinal fluid tau protein in differential diagnosis of Alzheimer's disease and vascular dementia in Chinese population: a meta-analysis. Am J Alzheimers Dis Other Demen. 2014;29(2):116-122. doi:10.1177/ 1533317513507374

34. Shoji M, Matsubara E, Murakami T, et al. Cerebrospinal fluid tau in dementia disorders: a large scale multicenter study by a Japanese study group. Neurobiol Aging. 2002;23(3):363-370. doi:10.1016/ S0197-4580(01)00309-8

35. Ceccaldi M, Jonveaux T, Verger A, et al. Added value of (18) F-florbetaben amyloid PET in the diagnostic workup of most complex patients with dementia in France: a naturalistic study. Alzheimers Dement. 2018;14(3):293-305. doi:10.1016/j.jalz.2017.09.009

36. Sonnen JA, Postupna N, Larson EB, et al. Pathologic correlates of dementia in individuals with Lewy body disease. Brain Pathol. 2010;20(3):654-659. doi:10.1111/j.1750-3639.2009.00371.x

37. Reesink FE, Lemstra AW, van Dijk KD, et al. CSF $\alpha$-synuclein does not discriminate dementia with Lewy bodies from Alzheimer's disease. J Alzheimers Dis. 2010;22(1):87-95. doi:10.3233/JAD-2010100186

38. Bellomo G, Paolini Paoletti F, Chipi E, et al. A/T/(N) profile in cerebrospinal fluid of Parkinson's disease with/without cognitive impairment and dementia with lewy bodies. Diagnostics. 2020;10 (12).

39. Bigio EH, Mishra M, Hatanpaa KJ, et al. TDP-43 pathology in primary progressive aphasia and frontotemporal dementia with pathologic Alzheimer disease. Acta Neuropathologica. 2010;120(1):43-54. doi:10.1007/s00401-010-0681-2

40. Josephs KA, Petersen RC, Knopman DS, et al. Clinicopathologic analysis of frontotemporal and corticobasal degenerations and PSP. Neurology. 2006;66(1):41-48. doi:10.1212/01.wnl.0000191307. 69661.c3

41. Le Bastard N, Martin JJ, Vanmechelen E, Vanderstichele H, De Deyn PP, Engelborghs S. Added diagnostic value of CSF biomarkers in differential dementia diagnosis. Neurobiol Aging. 2010;31 (11):1867-1876. doi:10.1016/j.neurobiolaging.2008.10.017

42. Biscetti L, Salvadori N, Farotti L, et al. The added value of A $\beta 42 /$ A $\beta 40$ in the CSF signature for routine diagnostics of Alzheimer's disease. Clin Chim Acta. 2019;494:71-73. doi:10.1016/j. cca.2019.03.001 


\section{Publish your work in this journal}

Clinical Interventions in Aging is an international, peer-reviewed journal focusing on evidence-based reports on the value or lack thereof of treatments intended to prevent or delay the onset of maladaptive correlates of aging in human beings. This journal is indexed on PubMed Central, MedLine, CAS, Scopus and the Elsevier
Bibliographic databases. The manuscript management system is completely online and includes a very quick and fair peer-review system, which is all easy to use. Visit http:/www.dovepress.com/ testimonials.php to read real quotes from published authors.

Submit your manuscript here: https://www.dovepress.com/clinical-interventions-in-aging-journal 